\title{
PERFIL EPIDEMIOLÓGICO DOS CASOS DE INTOXICAÇÃO DOMISSANITÁRIA NO PÚBLICO INFANTOJUVENIL
}

\section{EPIDEMIOLOGICAL PROFILE OF HOUSEHOLD INTOXICATION CASES IN THE INFANTOJUVENILE PUBLIC}

\author{
Maria Karoline Santos Lima ${ }^{1}$ \\ Hevillyn Cecília Ventura Barbosa ${ }^{2}$ \\ Josivan Soares Alves Junior ${ }^{3}$
}

\begin{abstract}
RESUMO: As intoxicações sejam elas ocupacionais, acidentais ou autoextermínio, constitui atualmente uma problemática a ser evidenciada pela saúde pública devido sua importância epidemiológica e social em decorrência dos crescentes números de notificação, sobretudo no público pediátrico. Objetivo: Posto isto, os objetivos do presente estudo é de revelar a partir do Sistema de Informações e agravos - SINAN os casos de intoxicações domissanitária em pacientes pediátricos, revelar o perfil sociodemográfico e epidemiológico do paciente acometido por intoxicação domissanitária, identificar as principais causas e circunstâncias de intoxicação por saneantes, bem como apresentar asprincipais condutas realizadas no atendimento a pacientes pediátricos vítimas destas substâncias com base na literatura. Método: Trata-se de um estudo transversal, retrospectivo e descritivo com abordagem quantitativa pautado em uma análise do Sistema de Informações e Agravos - SINAN e com base em manuscritos de bases de dados Scielo, Pubmed e Google acadêmico. Resultados: Portanto, que as intoxicações em público infantojuvenil reduz de acordo com o desenvolvimento dos indivíduos e que a faixa etária entre 1 a 4 anos são mais vulneráveis devido as peculiaridades própria da idade, além do gênero mais atingido ser o masculino e de característica acidental. Conclusão: Nisso, pode-se dizer que os óbitos devido às intoxicações por saneantes dependem da quantidade ingerida, bem como conduta realizada para reversão do caso e que apesar dos índices baixos de letalidade por uso indevido de domissanitário, não se isenta os malefícios causados pela exposição ao saneante que pode ofertar, em longo prazo, problemas respiratórios o ressaltando ainda mais a necessidade de implantação de medidas preventivas.
\end{abstract}

Palavras chave: Enfermagem. Saúde Integral. Toxicologia.

\footnotetext{
${ }^{1}$ Acadêmica de enfermagem pelo Centro Universitário - UNIFACISA. Campina Grande - PB.

${ }^{2}$ Acadêmica de enfermagem pelo Centro Universitário - UNIFACISA. Campina Grande - PB.

${ }^{3}$ Mestre em enfermagem, docente do Centro Universitário - UNIFACISA. Campina Grande - PB.
} 
ABSTRACT: Poisoning, whether occupational, accidental or self-extermination, is currently a problem to be highlighted by public health due to its epidemiological and social importance due to the increasing numbers of reports, especially in the pediatric public. Objective: That said, the objectives of the present study are to reveal, from the Information and Diseases System - SINAN, cases of domestic food poisoning in pediatric patients, to reveal the sociodemographic and epidemiological profile of the patient affected by domestic food poisoning, to identify the main causes and circumstances of sanitizing intoxication, as well as presenting the main conducts performed in the care of pediatric patients victims of these substances based on the literature. Method: This is a cross-sectional, retrospective and descriptive study with a quantitative approach based on an analysis of the Information and Diseases System - SINAN and based on manuscripts from Scielo, Pubmed and Google academic databases. Results: Therefore, that intoxications in children and adolescents reduces according to the development of individuals and that the age group between 1 to 4 years is more vulnerable due to the peculiarities of their age, in addition to the gender most affected being the male and of accidental characteristic. Conclusion: In this regard, it can be said that deaths due to intoxications by sanitizers depend on the amount ingested, as well as conduct performed to reverse the case and that despite the low rates of lethality due to the misuse of household cleaning products, the harm caused by exposure to sanitizing agent that can offer respiratory problems in the long term, further emphasizing the need to implement preventive measures

Keywords: Nursing. Comprehensive Health Care. Toxicology. 


\section{INTRODUÇÃO}

Consideradas um problema de saúde pública de cunho mundial, as intoxicações também existem diferenças sociais, geográficas, econômicas e culturais o que determina e difere os perfis em cada país. Diante dos mais de 12 milhões de produtos químicos, menos de 3.000 causam a grande parte das intoxicações acidentais ou de autoextermínio. Sustâncias ingeridas em grandes quantidades determinam o evento de exposição ou intoxicação da vítima podendo ser através de drogas, produtos domésticos, plantas, produtos agrícolas, químicos ou alimentícias. (ZAMBOLIN et al., 2008).

A intoxicação exógena pode apresentar-se pela introdução do agente tóxico em variadas vias, sendo as mais evidenciadas e de importância clínica: a respiratória, em que se mostra como porta de entrada para inalação de gases; a via cutânea em que é de fácil disseminação de conteúdo tóxico na pele; e a via oral ou digestiva que representa maior risco à saúde devido a ingestão determinada, intencional e em casos de criança, acidental (SILVA, 2018).

De acordo com Corrêa (2005) produtos de limpeza (cleaningproducts) ou também conhecidos "Domissanitário", é o nome genérico empregado no Brasil atribuído a elementos anti-infecções, desinfetantes e higienizastes. Os fabricantes classificam os saneantes de acordo com sua utilidade, sendo estes lava louças, limpadores de superfície, alvejantes, purificadores de ar, polidores, produtos de higiene pessoal (repelentes, inseticidas, raticidas e outros).

Sendo assim, a indústria publicitária, mormente os ressaltam pelas suas funções de poder de higienização e desinfecção sobre micróbios e microorganismos oportunistas que se dispõe sorrateiramente em âmbitos domésticos. A ANVISA, Agência Nacional de Vigilância Sanitária, classifica os saneantes de acordo com o gerenciamento de risco sendo analisados pela toxicidade da substância e concentração do produto; finalidade deuso; condições de uso; ocorrência de eventos 
adversos anteriores; população provavelmente exposta e formas de apresentação. (ANVISA, 2010).

Os saneantes são classificados em risco 1 quando possuem DL50 para ratos superior a $2000 \mathrm{mg} / \mathrm{kg}$ de peso corpóreo para produtos líquidos; e superior a $500 \mathrm{mg} / \mathrm{kg}$ de peso corpóreo para produtos sólidos; tenha Ph em forma pura, seja mantido em temperatura de $25^{\circ} \mathrm{C}$ e não apresentem corrosividade, ação microbiana, desinfetante e não contenha na formulação ácidos inorgânicos. Onde por outro lado, classifica-se como Risco 2 materiais semelhantes aos de risco 1, porém que apresentem corrosividade, ação microbiana, desinfectante e que contenha ácidos inorgânicos, a exemplo: Fluorídico, sulfúrico, nitrito (ANVISA ,2010).

Portanto, Fook (2013) em análise científica afirma que as exposições tóxicas a produtos domissanitários são comumente associadas a mulheres adultas e, sobretudo, e em maiores casos e agravos, em crianças menores de cinco anos. Visto que, no Brasil, os acidentes de alta toxicidade de causas não intencionais, constituem a principal causa de atendimento e internações em emergências pediátricas.

O uso de materiais saneantes domissanitários está regulamentado pela Agência Nacional de Vigilância Sanitária (ANVISA) de acordo com as resoluções 913 de 25 de junho de 2001, a resolução 184 de 22 de Outubro de 2001; e a RDC n³5 de 03 de junho de 2008, além da RDC n²25 de 25 de agosto de 2004 e as portarias n 327 de 30 de julho de 1997 e Portaria n 10 de 15 de setembro de 1980. (PINHO, 2014).

As intoxicações domissanitárias são caracterizadas pelo uso exacerbado, de modo acidental ou de autoextermínio, de substâncias destinadas a higienização ou desinfecção de ambientes coletivos ou públicos no tratamento de água ou em lugares de uso rotineiro. Pode-se incluir como saneantes os sabões e detergentes; desinfetantes; agentes de limpeza; inseticidas domésticos; raticidas e repelentes. (OGA et al., ANO).

De acordo com Mendonça (2015) o grande avanço tecnológico no século XX propiciou as indústria a se desenvolver e a ciência ter avanços consideráveis o que resultou no surgimento de novas substâncias tóxicas que são expostas no mercado para acesso da população sem na maioria das vezes ser informado os riscos e 
formas adequadas para seu uso e armazenamento. Isto posto, sabe-se que nos últimos anos a diversidade de produtos químicos tem sido resultado de maior exposição e, por conseguinte maiores acidentes não intencionais fazendo com que as intoxicações se tornassem um agravo de importância para saúde pública.

Alguns destes produtos se diferem em relação a finalidade de uso e os graus de toxicidade ao organismo humano. Substâncias como amaciantes, desinfetantes e desodorizantes possuem formulações que contém tensoativos catiônicos. Já nos detergentes, limpadores multiuso, bem como substâncias corrosivas como hidróxido de sódio têm em sua composição tensoativo aniônico e não aniônico que auxiliam no processo de limpeza do ambiente.

De acordo com Fook (2013) as intoxicações decorrentes do uso de saneantes são relativamente comuns para mulheres adultas e crianças, destacando o público infantil, em que os acidentes tóxicos, sobretudo, os de características não intencionais, constituia principal causa de atendimento de emergência pediátrica.

Isto posto, de acordo com Presgrave (2007), a faixa etária mais acometida é atéos 5 anos, fase característica do crescimento e desenvolvimento da criança, visto que com o início da locomoção, o desenvolvimento físico e de personalidade da criança e uma curiosidade em explorar o ambiente é um comportamento denominado "fase oral", em que a criança tende a levar tudo que está suscetível no ambiente a sua cavidade oral, o que se associa ao fato de ser um determinante destes acidentes nesta faixa etária.

Nisto, a Sociedade Brasileira de Pediatria (SBP) afirma que o público infantil torna-se vulnerável as intoxicações por saneantes devido a sua imaturidade física e mental, a inexperiência e incapacidade para proatividade em obstar-se de situações que Ihes oferte perigo, a curiosidade de realizar tarefas e o modo tendencioso de repetir comportamentos predispõe a criança a acidentes relacionados aos domissanitários. (TAVARES, 2013).

Vale salientar, que de acordo com os dados do sistema de informações tóxico farmacológicas - SINITOX (2015) as principais vítimas de envenenamento de modo acidental são crianças menores de 4 anos, visto que, nos últimos dez anos, cerca de $20 \%$ dos casos notificados pelo sistema referia-se a este público, fato que evidencia 
a necessidade de disseminação de conhecimento acerca da temática. (OLIVEIRA, 2017).

De acordo com o autor supracitado, a intoxicação é um evento comum e de importância enfatizada pelo envolvimento anual de dois milhões de crianças americanas com idade inferior a cinco anos. EMILIO (2000) enfatiza que as intoxicações neste público são evitáveis e que há necessidade de medidas públicas para prevenção de agravos nestes casos, visto que as crianças de 1 a 2 anos, pelo seu grau de desenvolvimento cognitivo age restritamente nas áreas da cozinha e lavanderia, propiciando ao uso de materiais para higienização doméstica.

É também na faixa etária entre 1 a 4 anos que as crianças desenvolvem habilidades para conseguir abrir mais facilmente a maioria dos recipientes e embalagens. A maior mobilidade os oferta a possibilidade de acesso a locais onde as famílias armazenam objetos e substâncias que constituem uma ameaça. Ademais, os responsáveis, devido ao processo de crescimento infantil, tendem a subestimar a capacidade das mesmas de conseguirem desenvolver determinadas ações. (LOURENÇO, 2008).

Dessa forma, com a proposta de desenvolver um sistema de informações que fosse essencial no trabalho da vigilância epidemiológica e subsidiasse no processo de controle de doenças, gestão de serviços, da prevenção de agravos e orientação sobre os mais incidentes casos de patologias que acometem a sociedade foi criado os sistemas de informações e agravos que se adapta a um meio extremamente mutável e de avanços tecnológicos rápidos (ARAÚJO; SILVA, 2015).

\section{MÉTODO}

Trata-se de um estudo transversal, retrospectivo e descritivo com abordagem quantitativa pautado em uma análise do Sistema de Informações e Agravos SINAN. Fora utilizado o site do Sistema de Notificações e Agravos - SINAN de endereço eletrônico:http://portalsinan.saude.gov.br/dados-epidemiologicos-sinan, contendo informações sobre todos os estados, municípios e regiões do Brasil, 
sendo evidenciado: Município de notificação; Faixa etária; Raça; Sexo; UF de exposição; agente tóxico; circunstância do agravo; tipo de exposição; classificação final; critério de confirmação;bem como a evolução do caso.

Ademais, como subsídio para disposição das informações analizadas e na apresentação dos resultados obtidos fora utilizado o programa Microsoft Office Excel 2007 para criação de tabelas e realização dos calculos de porcentagem dos dados.

\section{RESULTADOS E DISCUSSÃO}

Nos Três estados do nordeste (Paraíba, Rio grande do Norte, Pernambuco) foram totalizados 186 casos de intoxicação por domissanitários em crianças com faixa etária menores de 1 ano nos anos de 2010 a 2017 representando 4,27\% das intoxicações no público em geral, sendo na Paraíba 3,35\% ( $n=24)$, no Rio Grande do Norte 3,31\% ( $n=11)$ casos e em Pernambuco 4,53\% $(n=151)$ crianças intoxicadas por saneantes.

Tabela 1: Dados SINAWEB.

\begin{tabular}{cccccccccc}
\hline & \multicolumn{3}{c}{$>$ 1 ANO } & \multicolumn{3}{c}{ < ANOS } & \multicolumn{3}{c}{ 10-14 ANOS } \\
\hline & PB & RN & PE & PB & RN & PE & PB & RN & PE \\
2010 & 0 & 0 & 6 & 0 & 1 & 64 & 0 & 0 & 9 \\
2011 & 1 & 2 & 5 & 9 & 11 & 115 & 1 & 2 & 15 \\
2012 & 4 & 1 & 12 & 31 & 3 & 178 & 4 & 1 & 18 \\
2013 & 3 & 1 & 22 & 43 & 14 & 192 & 6 & 1 & 21 \\
2014 & 5 & 2 & 21 & 44 & 23 & 189 & 8 & 2 & 23 \\
2015 & 4 & 1 & 29 & 53 & 21 & 225 & 3 & 1 & 21 \\
2016 & 2 & 1 & 30 & 50 & 16 & 279 & 2 & 0 & 21 \\
2017 & 5 & 3 & 26 & 69 & 23 & 279 & 2 & 3 & 15 \\
Total & 24 & 11 & 151 & 299 & 112 & 1.521 & 26 & 10 & 143 \\
\hline Fotal
\end{tabular}

Fonte: Ministério da Saúde.

Todavia, Vilaça (2018) ao enfatizar em seus estudos que as relações entre fatores determinantes e desfechos no que tange infância e adolescência se modificam emdecorrência do local estudo devido ao acesso aos serviços de saúde e 
o tipo de agente tóxico utilizados, pode-se observar que, apesar da discrepância populacional, bem como, o acesso aos centros de saúde especializados e as possíveis subnotificações, os índices de intoxicação por saneantes em menores de um ano se mantiveram semelhantes nos três estados, o que implica dizer que as intoxicações acidentais nem sempre aumentam ou diminuem de acordo com os cenários e regiões, mas que caminham de acordo com a quantidade de pessoas que habitam em uma determinada região.

No público infantil de 1 a 4 anos, se observou nos dados do Sistema de Informação e Notificação de agravos - SINAN, que representa o maior número de intoxicações por saneantes, sendo $44,40 \%$ do total de intoxicações nos três estados. O estado da Paraíba do total dos 716 casos de intoxicação na população, 41,76\% foram por saneantes em crianças de 1-4 anos, enquanto no Rio grande do Norte dos 305 casos registrados $36,72 \%(n=112)$ foram neste público, e em Pernambuco foi representado por45,68\% $(n=1.541)$.

Em complemento, os dados do SINAN deixam expressos que mais de $40 \%$ dos casos de intoxicação por saneantes são no público infantil na faixa etária entre 1-4 anos. Nisso, as crianças com idade menor de quatro anos são mais vulneráveis as intoxicações devido à fase oral, explicado por Freud que afirma que a boca propicia a criança o conhecimento de mundo a sua volta, atrelado a isto, as crianças possuem um comportamento peculiar da idade, a curiosidade e caráter exploratório faz com que os mesmos tenham comportamentos que os exponham a agentes tóxicos ao seu alcance. (DOMINGOS et al., 2016; COUTO, 2017).

De acordo com a relação do gênero com as intoxicações domissanitárias ficou evidenciado em crianças do sexo masculino tem maiores índices de intoxicação sendo responsável por 53,51\% $(n=160)$ na Paraíba; $57,14(n=64)$ no Rio Grande do Norte e 57,07\% ( $n=868)$ no estado de Pernambuco. A OMS afirma que, os meninos sofrem comas intoxicação com mais freqüência que o público feminino, tal evento se explica devidoa diferença na socialização. A sociedade tem uma maior predisposição a educar os meninos sob vigilância menor quando comparado às meninas. Somado a isto, o fato dos meninos serem mais ávidos por exploração e buscarem brincadeiras mais arriscadas auxiliam na maior probabilidade de contato com os agentes tóxicos. (DOMINGOS et al., 2016). 
Isto posto, Ladeira (2016) em sua pesquisa ressalta que as intoxicações no público menor de quatro anos decorre por diversas ações, o que inclui a produção industrial, a legislação de comercialização dos produtos de uso doméstico, a regulação sanitária, o armazenamento dessas substâncias de modo inadequado, bem como, a falta de supervisão de crianças em âmbito doméstico.

Tais comportamentos fazem com que as intoxicações sejam caracterizadas em sua maioria como acidentais neste público. Portanto, na Paraíba 94,31\% ( $n=282)$ foram de causa acidental e 5,35\% tiveram outras causas; no Rio Grande do Norte $94,64 \%$ $(n=106)$ acidental e 5,36\% outros; enquanto em Pernambuco 90,93\% ( $n=1.383)$ acidental e de outras causam apenas $8,94 \%$. Nisso, pode-se observar que há uma predominância dos casos acidentes em comparação com as outras circunstâncias que levam uma criança nesta faixa etária a ser exposto aos saneantes.

Nesta esteira de raciocínio, o público entre 10 a 14 anos é pouco enfatizado na literatura visto que, observa-se que as intoxicações de características acidentais são mais prevalentes na faixa etária pediatria e que diminuem sua incidência com o decorrer do desenvolvimento emocional e cognitivo do indivíduos (OLIVEIRA, 2014). A Paraíba em 2010 a 2017 apresentou 53\% (n=14) dos seus casos acidentais neste público e 38\% ( $n=10)$ autoexterminio, no Rio Grande do Norte 40\% (n=04) acidental e $20 \%(n=02)$ tentativas de suicídio seguindo com $40 \%(n=04)$ outras causas, já em Pernambuco 53,85\% ( $n=77)$ dos casos foram acidentais neste público e $27 \%(n=39)$ foram tentativas de suicídio. Ainda assim, apesar dos casos reduzidos nesta faixa etária evidencia-se um crescente índice de intoxicações nos etária nos últimos anos. De acordo com o Manual de Toxicologia clínica (2017) o diagnóstico das intoxicações por saneantes dependem da substância a qual o individuo foi exposto. Todavia, a determinação de um evento de intoxicação é decorrente na análise dos sinais e sintomas clínicos, anamnese e história de exposição apresentados pela vítima. Alguns exames complementares servem de auxílio no diagnóstico e prognóstico, a exemplo: hemograma, eletrólitos, glicose, gasometria arterial, Raio X, endoscopia, ECG, entre outros. Os exames laboratoriais solicitados dependem da substância ingerida e da sintomatologia do paciente.

Na pesquisa realizada nos três estados do nordeste pode-se observar que os casos de intoxicação domissanitária são diagnosticados predominantemente pelos sinais e 
sintomas clínicos. A Paraíba apresentou 57,40\% ( $n=411)$ como critério de confirmação apenas pela clínica apresentada, bem como Rio Grande do Norte $71,80 \%(n=219)$ e Pernambuco $67 \%(n=2.231)$ dos casos foram diagnosticados pelos sintomas apresentados.

\section{CONCLUSÃO}

Conclui-se, portanto, que as intoxicações em público infantojuvenil reduz de acordo com o desenvolvimento dos indivíduos e que a faixa etária entre 1 a 4 anos são mais vulneráveis devido as peculiaridades própria da idade. Nisso, pode-se dizer que os óbitos devido as intoxicações por saneantes dependem da quantidade ingerida, bem como conduta realizada para reversão do caso e que apesar dos índices baixos de letalidade por uso indevido de domissanitário não isenta os malefícios causados pela exposição aos saneantes que podem ofertar em longo prazo alergias crônicas e outros problemas respiratório o que ressalta ainda mais a necessidade de implantação demedidas preventivas.

A constatação de que os maiores índices de intoxicação por domissanitários ressaltam a importância da utilização da medida de embalagem especial de proteção à criança - EEPC em que diz em sua lei $n^{\circ} 4$. 841-A/94 que medicamentos e produtos químicos de uso doméstico que apresentem um potencial de risco à saúde deverão ser confeccionados de modo que dificulte a abertura da embalagem pela criança, essa lei implantada nos Estados Unidos e Canadá resultaram em uma diminuição de 35\% dos casos de intoxicação por esses agentes. (FOOK, 2013; DINIZ 2014). 


\section{REFERÊNCIAS BIBLIOGRÁFICAS}

ALBUQUERQUE, P. C. C. D. et al. Health information systems and pesticide poisoning at Pernambuco. Revista Brasileira de Epidemiologia, v. 18, p. 666-678, 2015. ISSN 1415$790 X$.

AMORIM, M. L. P.; MELLO, M. J. G.; SIQUEIRA, M. T. Intoxicações em crianças e adolescentes notificados em um centro de toxicologia no nordeste do Brasil. Rev. Bras. Saude Mater. Infant., Recife, v. 17, n. 4, p. 765-772, $2017 . \quad$ Disponível em: $<$ http://www.scielo.br/scielo.php?script=sci_arttext\&pid=S1519-

38292017000400765\&lng=en\&nrm=iso>. Acesso em 03 de Mai de 2020. http://dx.doi.org/10.1590/1806-93042017000400009.

ARAÚJO, M. M. P; SILVA, C, G. A importância do sistema de informação de agravos de notificação - SINAN para a vigilância epidemiológica do Piauí. Revista Interdisciplinar Ciências e Saúde, v. 2, n.3, 2015.

FOOK, S. M. L. et al. Avaliação das intoxicações por domissanitários em uma cidade do Nordeste do Brasil. Cad. Saúde Pública, Rio de Janeiro, v. 29, n. 5, p. 1041-1045, 2013. Disponivel em: <http://www.scielo.br/scielo.php?script=sci_arttext\&pid=S0102311X 2013000500021\&lng=en\&nrm=iso>. Accesso em: 03 de Mai de 2020. https://doi.org/10.1590/S0102-311X2013000500021.

GALVÃO, P. R.S.; FERREIRA, A. T; MACIEL, M. G. G.; ALMEIDA, R. P.; HINDERS, D.; SCHREUDER, P. A. et al. Uma avaliação do sistema de informação SINAN usado no Programa de Controle de Hanseníase no estado do Pernambuco, Brasil. Cad. Saude Coletiva, v.17, n. 1, p. 87-102, 2009.

LAGUARDIA, J. et al. Sistema de informação de agravos de notificação em saúde (Sinan): desafios no desenvolvimento de um sistema de informação em saúde. Epidemiol. Serv. Saúde, Brasília v.13, n3, p.135-146, set 2004. Disponível em: $<$ http://scielo.iec.gov.br/scielo.php?script=sci_arttext\&pid=S167949742004000300002\&lng=pt \&nrm=iso $>$. Acesso em 08 de abr. de 2020.

LAGUARDIA, J. et al. Sistema de informação de agravos de notificação em saúde (Sinan): desafios no desenvolvimento de um sistema de informação em saúde. Epidemiol. Serv. Saúde, Brasília v.13, n3, p.135-146, set 2004.

MAIA, D. A. B. et al. Avaliação da implantação do Sistema de Informação de Agravos de Notificação em Pernambuco, 2014. Epidemiol. Serv. Saúde, Brasília, v. 28, n. 1, 2014.

MARIN-LEON, L.; BARROS, M. B A. Mortes por suicídio: diferenças de gênero e nível socioeconômico. Rev. SaúdePública, São Paulo, v. 37, n. 3, p. 357-363, June 2003. Disponível em: <http://www.scielo.br/scielo.php?script=sci_arttext\&pid=S003489102003000300 015\&lng=en\&nrm=iso>. Acesso em: 03 de Mai de 2020. https://doi.org/10.1590/S003489102003000300015 .

MENDONÇA, D. H. Intoxicaçoes Exógenas Agudas em Crianças e Adolescentes em um Hospital Público na Bahia. 2015. 87 f. Tese (Mestrado) - Curso de Farmácia, Escola Bahiana de Medicina e Saúde Pública. Programa de Pós-graduação em Tecnologias em Saúde, Salvador - Bahia, Cap. 1, 2015.

PRESGRAVE, R. F. Avaliação das intoxicações acidentais humanas causadas por produtos saneantes domissanitários como subsídio para ações de vigilância sanitária. 
2007. 160 f. Tese (Doutorado em Vigilância Sanitária) - Instituto Nacional de Controle de Qualidade em Saúde, Fundação Oswaldo Cruz, Rio de Janeiro, 2007.

VILACA, L.; VOLPE, F. M.; LADEIRA, R. M. Intoxicações Exógenas Acidentais em Crianças e Adolescentes Atendidos em um Serviço de Toxicologia de Referência de um Hospital de Emergência Brasileiro. Rev. paul. pediatr., São Paulo, v. 38, e2018096, 2020. Disponível em: <http://www.scielo.br/scielo.php?script=sci_arttext\&pid=S01030 $5822020000100401 \&$ Ing=en\&nrmso>. Acesso em: 03 de Mai de 2020.

VILACA, L; VOLPE, F, M; LADEIRA, R, M. Intoxicações Exógenas Acidentais em Crianças e Adolescentes Atendidos em um Serviço de Toxicologia de Referência de um Hospital de Emergência Brasileiro. Rev. paul. pediatria., São Paulo, v. 38, 2020. 